\title{
LO INDECIBLE EN EL TRACTATUS
}

LUIS VILLORO

Universidad Autónoma Metropolitana

La mayoría de los comentadores del Tractatus Logico-Philosophicus lo han visto como una obra que trata fundamentalmente de lógica. La estructura del libro se prestaba, sin duda, a esa interpretación: las cuestiones sobre el lenguaje, su forma lógica y su relación con el mundo abarcan casi toda la obra; la parte final que contiene los enunciados más importantes sobre temas "éticos" y "metafísicos", comprende apenas unos pocos aforismos enigmáticos, difíciles de comprender. Tanto la interpretación positivista como la de Bertrand Russell hicieron a un lado, con molestia y cierto desdén, la parte que trata de lo "indecible", como si fuera un añadido incongruente con el resto. Russell y Ramsey ejemplifican dos formas diferentes de esa misma actitud. El primero confiesa su intelectual discomfort ante el misticismo de Wittgenstein y adelanta una hipótesis lógica que evitara acudir a él. Lo que no puede ser dicho en un lenguaje, según esa hipótesis, podría ser dicho en otro lenguaje con una estructura distinta y así sucesivamente, en una jerarquía sin límite de niveles de lenguaje. ${ }^{1}$ Así, la existencia de lo indecible a la que apunta todo el Tractatus debe ser conjurada con una hipótesis ad hoc: se trata de un problema que hay que resolver.

La via de Ramsey es distinta pero revela un desinterés semejante por esa parte del libro. Según Ramsey, Wittgenstein debió haber tomado en serio su tesis sobre lo indecible

1 Tractatus Logico-Philosophicus, Introducción, Routledge and Kegan Paul, New York, 1961, p. XXII. 
y no haber intentado comunicar nada sobre ello. Si todo lo que rebasa las proposiciones científicas carece de sentido, Wittgenstein debió atenerse a ello y no pretender darle importancia a un sinsentido. ${ }^{2}$ Una postura semejante es tomada por autores posteriores. Es sintomático que un comentario tan extenso como el de Marx Black, que consta de 451 páginas, dedique sólo 15 a las entradas finales del Tractatus."

Sin embargo, esas entradas pretenden mostrar el sentido total de la obra. No por casualidad están colocadas al final del libro, como su consecuencia. La penúltima entrada del Tractatus, antes del silencio, (6.54) da la clave de su lectura. La obra pretende conducir a ver el mundo "correctamente" (richtig), pero para ello es menester "superar" las proposiciones del Tractatus. Una lectura puramente lógica no hace esa operación. El propio Wittgenstein fue explícito al respecto y no consideró su escrito como una obra de lógica. "El objetivo del libro es ético —escribía a Ficker- [...] Mi obra consta de dos partes: la que aquí se presenta, más todo lo que no he escrito. $Y$ es precisamente esta segunda parte la que es importante. Mi libro traza límites a la esfera de lo ético, desde dentro por así decirlo, y estoy convencido de que ésta es la única manera rigurosa de trazar esos límites." "

Tienen razón Janik y Toulmin al pedir una relectura del Tractatus en consonancia con esa opinión, aunque ellos mismos no hayan acertado a dárnosla. No sólo cambia el sentido de la obra si se la ve bajo ese aspecto sino que sólo entonces puede recibirse su mensaje, lo que realmente quiso comunicar su autor. Y ese mensaje, creía Wittgenstein, es de mucho mayor interés que todas las doctrinas lógicas incluidas en la obra. Que lo verdaderamente importante del Tractatus

2 The Foundations of Mathematics, Routledge and Kegan Paul, London, 1961, p. 263.

3 M. Black, A Companion to Wittgenstein's Tractatus, Cambridge Univ. Press, 1964.

4 Citado por A. Janik y S. Toulmin, Wittgenstein's Vienna, Simon and Schuster, New York, 1973, p. 192. 
no es lo que se dice en él, sino lo que no puede decirse en él y sólo se comunica después de haber comprendido que no puede decirse.

El Tractatus tiene por fin establecer claramente los límites del lenguaje con sentido. "Así, el libro quiere poner un límite al pensamiento, o más bien, no al pensamiento, sino a la expresión de los pensamientos" ("Prefacio", p. 3). El análisis del lenguaje significativo conduce a establecer condiciones últimas que lo hacen posible. Mas esas condiciones no forman parte, a su vez, del lenguaje significativo, no están "dentro" de él. Los límites del lenguaje no pueden ser "dichos" como cualquier hecho describible por el lenguaje. La obra conduce así del análisis de lo decible por el lenguaje a sus condiciones indecibles.

Pero "los límites de mi lenguaje se refieren (bedeuten) a los límites de mi mundo" (5.6). El análisis del lenguaje significativo conduce simultáneamente, de la consideración de los hechos describibles por el lenguaje, que componen el mundo, a la visión de sus límites indescriptibles. Todo el Tractatus conduce así a la visión del mundo como un "todo limitado".

Sólo entonces estoy en situación de tener una "visión correcta" del mundo. Por desgracia, para llegar a ella hay que superar una paradoja aparentemente incomprensible: quien habla de los límites del lenguaje o del mundo comete un sinsentido. El mensaje del Tractatus sólo puede captarse cuando se "entiende" que sus oraciones más importantes pretenden hablar de lo que no puede hablarse y carecen, por ende, de sentido. La paradoja puede expresarse también en otra forma. Las proposiciones del Tractatus deben comunicar algo si podemos entender que carecen de sentido. Las oraciones sin sentido deben poder comunicar algo si conducen a una visión "correcta" del mundo. La comprensión de esa paradoja es la única que puede darnos la clave del Tractatus.

Este artículo se planteará tres preguntas: 
¿Cómo conduce el Tractatus a la visión del mundo como un todo limitado?

¿Cómo es posible comunicar esa visión mediante sinsentidos?

¿Cuál es la visión "correcta" que hace posible esa co. municación?

\section{Las condiciones de lo decible}

Con la entrada 6.4 ("Todas las proposiciones tienen el mismo valor") se inicia la parte final del Tractatus que habla de lo indecible. Con la excepción de las entradas 5.6 a 5.641 (que tratan de los "límites" del lenguaje y del mundo y del "yo metafísico"), las proposiciones del libro habían hablado hasta ahora, tanto de la forma y componentes de un lenguaje figurativo y de lo figurado por él, como de las condiciones que hacen posible ese lenguaje. Las oraciones que van de 6.4 al final (más 5.6 a 5.641) pretenden hablar, en cambio, de la totalidad del mundo y del lenguaje, así como de lo que está "fuera" del mundo y "más allá" del lenguaje. ¿Cómo se llega a dar ese paso?

$\mathrm{EI}$ análisis del lenguaje figurativo muestra que éste supone ciertas condiciones que operan como sus límites y que son ellas mismas indecibles por el lenguaje figurativo.

En primer lugar, el análisis conduce a aceptar últimos elementos simples, ya no analizables, representados en las pro. posiciones por signos simples. Son los objetos. Tiene que admitirse objetos para que el lenguaje sea determinado. La existencia de objetos simples es una condición de todo lenguaje figurativo. Pero si los objetos son condición de lo decible ellos mismos no son decibles, esto es, no pueden ser representados por una figura (Bild). "Sólo puedo hablar de ellos, no puedo expresarlos ellos mismos. Una proposición sólo puede decir cómo es una cosa, no lo que es" (3.221).

5 Adopto la traducción de Bild por "figura", propuesta por Tierno Galván, que parece efectivamente la mejor. 
Es evidente que no puedo construir una figura que represente a un objeto, puesto que los objetos son los elementos que hacen posible que se construya cualquier figura. Y sólo es decible lo representable en figuras. Luego, los objetos son propiamente indecibles. Sólo pueden ser designados como "aquéllo" a que se refieren los signos simples, sólo pueden ser nombrados (3.221).

El objeto es el "esto" puro, el sustrato último del que puedan predicarse cualidades y que él mismo no puede predicarse de nada. Es pues la sustancia en un sentido aristotélico (2.021). ${ }^{6} \mathrm{Y}$ no puedo decir lo que sea la sustancia; como tal es indescriptible. Los objetos, sustancia del mundo, están supuestos en todos los hechos capaces de ser figurados por el lenguaje, pero ellos mismos no son hechos. Si el mundo es "todo lo que acontece" (1), o sea, la totalidad de los hechos (1.1), la sustancia no forma parte del mundo, porque no es ningún hecho. "La sustancia es lo que existe independientemente de lo que acontece" (2.024).

En este sentido, podemos decir que los objetos no forman parte del mundo pero, a la vez, son los elementos últimos que debo suponer en cualquier hecho del mundo. La sustancia no está "dentro" del mundo, como una de sus partes, pero establece el "límite" dentro del cual puede darse un mundo. Los objetos no pueden ser dichos en el lenguaje pero son la condición exigida para que cualquier proposición diga algo con sentido. Así, el análisis de lo decible conduce a últimos elementos indecibles; éstos señalan los supuestos bajo los cuales puede figurarse ("representarse", "pensarse") algo. El objeto es pues la primera manifestación de los límites del mundo y del lenguaje; se manifiesta como la existencia pura de "algo" indescriptible.

En segundo lugar, el análisis del lenguaje figurativo con-

- La sustancia es, para Aristóteles, por una parte, el sustrato concreto último de toda cualidad, el mero "esto aquí" particular, supuesto en cualquier atribución, por la otra, el sujeto que nunca puede ser predicado de nada (cfr. Metafísica, libro Delta, $1017^{\mathrm{b}}, 10$ ). 
duce a admitir la forma lógica como su condición a priori. Toda proposición supone lo que tiene en común con el hecho que figura: la forma lógica. Pero la forma lógica no puede ser ella misma figurada por el lenguaje. "Las proposiciones pueden representar toda la realidad, pero no pueden. representar lo que deben tener en común con la realidad para poder representarla: la forma lógica" (4.12). La forma lógica se muestra, sin embargo, en toda proposición con sentido (4.121). Al igual que el objeto, la forma lógica es indecible, sólo puede mostrarse en lo decible.

En todo uso significativo del lenguaje, en cualquier pensamiento se muestra, sin ser mencionado, su supuesto: los objetos, como sustratos últimos a que se refiere el lenguaje, y la forma lógica, como "espacio" en que se construye toda figura. Pero ese supuesto no es el último; supone, a su vez, una "experiencia" básica: la existencia, allí, del mundo. "La experiencia que necesitamos para entender la lógica no es que algo esté en tal o cual situación, sino que algo sea; pero esto no es una experiencia. La lógica es anterior a cualquier experiencia de que algo sea así. Es anterior al cómo [sea algo], pero no al que [algo sea]" (5.552). Y esa "experiencia", presupuesta en la lógica, es justamente la que Wittgenstein llama "mística". "Lo místico no es cómo sea el mundo, sino que el mundo sea" (6.44).

Así, bajo los supuestos lógicos del lenguaje debemos admitir otro supuesto, "de segundo nivel" por así decirlo, extralógico. Pues bien, todo el Tractatus conduce a la mostración de ese último supuesto. Veamos cómo.

\section{El mundo como un todo limitado}

6.4 es la primera entrada de la parte final que se ocupa de lo "místico", pero puede verse también como el resultado de las tesis establecidas inmediatamente antes. En las páginas precedentes, en efecto, se ha sentado:

1. La lógica es la condición a priori del mundo; esta condición sólo puede mostrarse en las proposiciones que hablan 
del mundo. "La lógica llena el mundo; los límites son también sus límites" (5.61). La lógica establece pues la "forma total", el "espacio" en el que puede ser el mundo.

2. Las leyes de la ciencia son reducibles a la lógica y no hay más necesidad que la necesidad lógica (6.37). Pero la necesidad lógica es tautológica y no describe ningún he: cho del mundo. Luego, el mundo, como totalidad de los hechos, es absolutamente contingente. Es un conjunto de hechos que podrían haber sido de cualquier otra manera o, simplemente, no haber sido. Por ello cualquier acontecimiento - como la salida del sol mañana - podría ser de otra manera (6.36311). "Todo lo que vemos podría ser diferente de lo que es" (5.634). Ni la ciencia, ni la lógica "explican" la existencia de los hechos del mundo (6.371).

3. Este mundo absolutamente contingente está ahí, presente, independientemente de que yo lo quiera o no, ajeno a mi voluntad (6.373).

Las proposiciones que anteceden a 6.4 establecen pues una consecuencia: la existencia del mundo como un todo limitado por el espacio lógico, absolutamente contingente en un doble sentido: podría haber sido de cualquier otro modo o no haber sido y es algo con lo que me encuentro, independientemente de mi querer. Estamos preparados para aceptar 6.4: todas las proposiciones de que puedo hablar son "equi-valentes" (gleichwertig), es decir, ninguna tiene más valor que otra. Porque el juicio de valor está excluido de ellas. El mundo contingente que se explaya en el espacio lógico es indiferente a toda apreciación de valor o disvalor, simplemente está ahí, sin más, como un hecho inexplicable. Pero decir que el mundo en el espacio lógico no comprende el valor es lo mismo que decir que cualquier cuestión sobre el valor (cualquier enunciado de la ética o de la estética, por ende) está "fuera del mundo" (6.41).

Si me refiero a algo "fuera" del mundo, contemplo el mundo con un "fuera" y un "dentro", es decir, con un $l i$ mite. La visión del mundo como un todo limitado no es pro- 
piamente una experiencia, porque no se refiere a un hecho que pueda estar en relación con otros hechos, se refiere a la circunstancia de que un hecho y todo conjunto de hechos sea... esté ahí como un simple hecho. Es una visión (Anschauung) y un sentimiento (Gefül) que no caen dentro del campo de lo representable ("figurable") por el lenguaje. "El sentimiento del mundo como un todo limitado es lo mistico" (6.45).

No es casual que Wittgenstein emplee la palabra "místico". La consideración del mundo como un acontecimiento inexplicable, gratuito, que hubiera podido no ser $\mathrm{y}$, sin embargo, es, causa un indescriptible asombro. Ahí está el mundo, como un milagro brotado de la nada. En lugar del vacío infinito, la presencia inefable del universo. No es sólo la perplejidad de la razón ante lo incomprensible para ella, es un sentimiento de pasmo, de estupor, ante lo extraño por excelencia: lo otro, la presencia misma del mundo.

Este sobrecogimiento ante lo otro es la base de toda experiencia religiosá genuina. No se trata, claro está, de la creencia en alguna entidad supramundana, dios o demonio, ni de la experiencia de alguna "hierofanía" particular en que se crea vislumbrar lo ultramundano, sino del sentimiento del universo como lo otro por excelencia, es decir, lo Sagrado. Rudolf Otto vio muy bien como el estupor ante lo Sagrado, experimentado como "misterio tremendo y fascinante", es la raíz de toda experiencia religiosa. ${ }^{\top}$ Porque el portento puro y simple no es tal o cual hecho que aconteza en el mundo, sino la existencia inexplicable del mundo. $\mathrm{Mu}$ chos milagros hay en el mundo -dice San Agustín- pero "el mayor milagro de todos es el mundo mismo". 0 pode. mos expresar lo mismo con Wittgenstein, a la inversa: el asombro ante la existencia del mundo es "la experiencia de ver el mundo como un milagro" (alsein Wunder.)

7 Das Heilige, Biederstein Verlag, München, 1947.

8 Civitas Dei, Lib. XXI, cap. IX.

"Wittgenstein's Lecture on Ethics", en Philosophical Review, LXXIV, 1965 , p. 11 . 
Esta podría ser también la raíz de la experiencia estética. Así pareció entenderlo, al menos, Wittgenstein: "El milagro artístico (das kunstlerische Wunder) es que el mundo exista. Que exista lo que existe." ${ }^{10} \mathrm{Y}$ podría ser también la raíz última de toda actitud ética. "Piensen, por ejemplo, en el asombro porque algo exista. Este asombro no puede ser expresado en forma de una pregunta, ni hay tampoco respuesta para él. Todo lo que podemos decir sobre él sólo puede ser, a priori, un sinsentido. Con todo, chocamos contra los límites del lenguaje. Esto también lo vio Kierkegaard y lo indicó, de un modo enteramente similar, como chocar contra la paradoja. Este chocar contra los límites del lenguaje es la ética." ${ }^{11}$ La religión, la estética, la ética, toda actitud dirigida hacia los valores absolutos, toda pregunta por el "sentido" del mundo y de la vida, choca contra los límites del lenguaje y se basa en la captación del mundo como portento inexplicable.

La existencia del mundo como un todo limitado es condición de todo lenguaje figurativo. Se muestra, por ende, en cualquier uso que hagamos del lenguaje conforme a reglas lógicas. Pero sólo suscita nuestro estupor cuando somos capaces de resentir, sin palabras, la silenciosa presencia del mundo como pura maravilla inexpresable. Por ello se acompaña de un sentimiento peculiar.

"Limitado" es, por supuesto, una manera metafórica (y, como veremos, "sin sentido") de hablar. Ver algo como limitado es contrastarlo con algo que estaría "afuera". Pero fuera del mundo estrictamente no hay nada. Porque el mundo es la totalidad de lo que acontece y no hay ningún hecho que no abarque. Ver el mundo como limitado es, pues, contrastarlo con lo que no es un hecho, con lo que no sucede. Sería verlo, por así decir, "en vilo" sobre la nada. Pero ésta es una manera sumamente oscura y confusa de expre-

10 Notebooks 1914-1916, Blackwell, Oxford, 1961, p. 86.

11 Ludwig Wittgenstein und der Wiener Kreis, B. F. MacGuinness ed., Blackwell, Oxford, 1967, p. 68. 
sar, con palabras que carecen de sentido, el sentimiento de la existencia del mundo como habiendo podido no ser. Sentir el mundo como un todo limitado es captarlo, sin pensamiento, como algo finito - fuera de lo cual no hay nadaque está ahí sin explicación.

Esta visión no corresponde al pensamiento ni cae en la esfera de la representación. Para tenerla hay que rebasar esa esfera y dejar que el mundo se ofrezca a nuestro sentimiento. Depende, pues, de la actitud que tengamos ante el mundo. Por ello corresponde a la voluntad. La manera como el mundo en su totalidad se muestra a cada quien depende de su elección de valores y de su actitud ante él. "Si la buena o la mala voluntad altera el mundo, sólo puede alterar los límites del mundo, no los hechos, no lo que puede ser expresado por el lenguaje. En suma, de ese modo el mundo debe convertirse en otro enteramente distinto. Debe, por así decirlo, ampliarse o reducirse (abnehmen oder zunehem) como un todo. El mundo del hombre dichoso es otro que el del desdichado" (6.43).

Cualquier hecho del mundo es independiente de la volun. tad (6.373) ; no puedo, con mi querer cambiar ningún acontecimiento. Sin embargo, mi vivencia del universo varía, de modo que puedo resentirlo como un mundo más o menos "Pleno", con mayor o menor sentido, según sea mi voluntad.

Por supuesto que por "voluntad" no entiende Wittgenstein el querer consciente, resultado de una deliberación racional. El término proviene de la filosofía de Schopenhauer, en la cual se opone a "representación". La voluntad se refiere a la esfera de impulsos, emociones, actitudes vitales que no pueden ser objeto de representación, que no caen, por ende, en el campo de lo decible en figuras ni de lo pensable (puesto que sólo lo que puede decirse puede pensarse).

Antes de callar, entre las proposiciones que dicen cómo es el mundo figurable por el lenguaje y el silencio exigido por lo indecible, Wittgenstein cede a la tentación de balbucir lo que, en rigor, no puede ser dicho. Ese balbuceo co- 
munica la visión del mundo a la que conduce el Tractatus. Pero esa visión global es la que subyace también a lo largo de toda la exposición del libro, constituye como un fondo sobre el cual se inscriben todas sus proposiciones. En efecto, la primera proposición, de la que derivan las demás, la supone: "El mundo es todo lo que acontece". Esta definición expresa la visión del mundo como un todo de hechos contingentes. La proposición 6.44 ("lo místico es que el mundo sea") se conecta con la proposición 1. Místico no es ver cómo es el mundo, es decir, verlo como un conjunto de cosas con cualidades y relaciones distintas, sino verlo en su pura existencia fáctica, como una "totalidad de hechos, no de cosas" (1.1).

La misma visión del mundo que concluye el Tractatus, lo antecede. El punto final es también el principio. La obra entera se inscribe sobre esa visión del mundo.

\section{Las oraciones sobre lo indecible}

Pero ¿cómo expresar esa visión del mundo? Las oraciones que traten de expresarla no podrán ser hechos en el mundo, luego, no podrán ser figuras de ningún hecho. Aquello que tratan de expresar tampoco es un hecho particular sino la totalidad de los hechos. ¿Cómo hablar de la totalidad si toda proposición es un hecho que figura un hecho?

Las oraciones que expresamente se refieren a lo indecible se encuentran principalmente en dos lugares: entre las entradas 5.6 y $5.641 \mathrm{y}$, en la parte final, de la 6.4 a la 6.522 . En términos muy generales podríamos agruparlas en tres tipos:

1. Oraciones negativas. Dicen que algo no está en el mundo, o bien que una pseudoproposición no forma parte del lenguaje. Son la mayoría.

Por ejemplo: "Si escribiera un libro intitulado 'El mundo tal como lo encuentro' ... del sujeto no podría hablarse en ese libro" (5.631). 
"El sujeto no pertenece al mundo" (5.632).

"Todas las proposiciones son equivalentes" (igual: "ninguna proposición tiene valor") (6.4).

"El sentido del mundo debe estar fuera del mundo"... "Lo que hace al mundo no-contingente no puede estar en el mundo" (6.41).

"Así, no puede haber tampoco proposiciones de la ética" (6.42).

"Es claro que la ética no puede expresarse" (6.421).

"Es imposible hablar de la voluntad como portadora de lo ético" (6.423).

"Dios no se revela en el mundo". (6.432).

"La muerte no es un acontecimiento en la vida" (6.4311).

"Si una respuesta no puede expresarse, tampoco la pregunta puede expresarse" (6.5).

Que el mundo exista es el "hecho" de que hay una totalidad de hechos. Pero ese "hecho" no es un acontecimiento de los que constituyen el mundo. En sentido estricto no tiene sentido, pues, llamarlo "hecho", porque cualquier hecho acaece en el mundo, pero el mundo mismo no acaece en nada. Lo místico ("que el mundo.sea") no se refiere, pues, a ningún acontecimiento del mundo.

Por otra parte, el valor y el sentido del mundo y de la vida, de que pretenden hablar los enunciados de la ética y de la estética, tampoco pueden encontrarse en hechos. La descripción exhaustiva de los componentes de los hechos nunca podrá incluir un valor. Este no es una cualidad fáctica que pueda describirse. Si describiéramos la totalidad de lo que acontece no encontraríamos, como un acontecimiento más, sentido o absurdo, bien o mal, belleza o fealdad. Si el mundo es la totalidad de los hechos en el espacio lógico y si en ellos no se encuentra el valor ni el sentido, no podemos decir que éstos estén en el mundo. Así, las oraciones negativas no describen nada sobre lo místico, sobre el valor o el sentido, sólo comunican lo que no son. Comunican que no son hechos, que están "fuera" del mundo. 
Por otra parte, los enunciados sobre el valor y el sentido no son proposiciones. Toda proposición es una figura (Bild) de hechos, una descripción de lo que acontece. Por ello toda proposición es indiferente al valor (6.4). El valor y el sentido de la vida no son hechos figurables en el lenguaje. Las oraciones en que se encuentran esos términos son, pues, pseudoproposiciones que no figuran ninguna realidad. Las oraciones negativas no dicen en qué consisten las pseudoproposiciones de la religión, la ética y la estética, sólo comunican lo que no son. Comunican que no forman parte del lenguaje con sentido.

Pero comunicar que lo místico no es en el mundo, no es afirmar que no exista. Las oraciones negativas que reseñamos presuponen la afirmación de la existencia de algo "fuera" del mundo y del lenguaje.

Ejemplos: "El sentido del mundo debe estar fuera del mundo" (6.41) afirma implícitamente que hay un "sentido del mundo".

"La ética es trascendental" (6.421) asevera que hay una ética.

"Cómo sea el mundo, es enteramente indiferente a lo más alto. Dios no se revela en el Mundo" (6.432) da por supuesto que existe "lo más alto", y así sucesivamente.

Aún, en una sola entrada, la existencia de lo místico está explícitamente afirmada: "Existe, de cualquier modo, lo indecible. Se muestra, es lo místico" (6.522). Esta oración podría igualmente expresarse en forma negativa: "Lo místico no es decible, se muestra."

2. Oraciones sobre el todo o sobre los límites del mundo o del lenguaje.

Ejemplos: "La lógica llena el mundo; los límites del mundo son también sus límites" (5.61).

"El sujeto ... es un límite del mundo". (5.632).

"Ver el mundo sub specie aeterni es verlo como un todo limitado" (6.45).

"Si la buena o mala voluntad altera el mundo, sólo pue- 
de alterar los límites del mundo, no los hechos"... “El mundo debe, por así decirlo, ampliarse o reducirse como un todo" (6.43).

"La ética es trascendental" (6.421).

Lo místico abarca aquello que no es un hecho figurable en el mundo pero que se muestra en la visión del mundo como un todo limitado. Al decir que está "fuera" del mun. do no puede entenderse que esté en algún "trasmundo o "supramundo", porque más allá del mundo no hay estrictamente nada. Usando una distinción de procedencia kantiana, podemos decir que lo místico no es trascendente al mundo, sino trascendental. "Trascendente" es lo que rebasa la experiencia posible y se supone que existe más allá de sus límites. Se refiere a la existencia de ciertas cosas o hechos no captables por la experiencia ni expresables en el lenguaje científico, pero que subsistirían en alguna región entitativa diferente de la experiencia. Esa trascendencia no cabe en la concepción del Tractatus. Lo "trascendental" en cambio, según Kant, no está propiamente "más allá" de toda experiencia, sino "más acá" de ella. Es el conjunto de condiciones que la hacen posible; éstas no son experimentables, porque no constituyen una parte de la experiencia, pero debo admitirlas para el todo de la experiencia.

De parecida manera, en Wittgenstein, la lógica es "trascendental" (6.13), porque es la condición de todo lenguaje capaz de figurar el mundo y, a su vez, no puede ser figu. rada por el lenguaje. Igualmente la visión del mundo como un todo limitado es condición de toda experiencia y no es, a su vez, una experiencia más. Lo místico no está, pues, "más allá" del mundo, sino que está presupuesto en cualquier experiencia del mundo.

La ética es, igual que la lógica, "trascendental" (6.421).$^{12}$ Por "ética" Wittgenstein entiende la investigación de "lo que es valioso o lo que es realmente importante", del "sen-

12 Cfr., también: "La lógica y la ética son condiciones del mundo", Notebooks, p. 77. 
tido de la vida, o lo que hace a la vida digna de ser vivida, o la manera correcta de vivir". ${ }^{13}$ Es una con la estética (6.421) y abarca, por ende, todo enunciado de valor. Ética y estética no son "trascendentes". En efecto, el valor y el sentido de la vida no forman parte de la experiencia de un hecho, pero tampoco se encuentran en otros "hechos" supramundanos, ni requieren de algún tipo extraordinario de experiencia dirigida a alguna región entitativa "sobrenatural". El valor y el sentido de la vida se muestran en la vida y en el mundo considerados como un todo. Son presupuestos generales de la experiencia del mundo. Igual que la lógica, la ética está presupuesta en la captación del mundo como un todo.

Si lo trascendental no está en el mundo ni tampoco en otra región supramadura, ¿dónde está? No está en ninguna parte. Corresponde al todo en cuanto todo. En lo trascendental está, pues, todo. Esto es lo que expresaría Wittgenstein con la palabra "límite". Los límites del mundo están determinados justamente por la lógica y corresponden a los trazados por el lenguaje figurativo $(5.61,5.62)$.

Por supuesto que el "límite" del mundo no puede entenderse como una frontera que separaría la totalidad de los hechos de algo fuera de ellos. Lo trascendental limita el mundo en el sentido de que establece las condiciones generales en que éste se da, sin las cuales no podría darse. La lógica determina los límites de lo decible porque señala el "espacio" en que puede formularse cualquier proposición. La ética determina la manera como el mundo puede presentarse como un todo ante la buena o la mala voluntad. Al alterarse la voluntad, ningún hecho del mundo cambia, pero la totalidad de los hechos se muestra con un aspecto distinto: "El mundo del hombre dichoso es otro que el del desdichado" (6.43). La vountad determina la manera global en que se da el mundo. Así, el círculo supone la circunferencia que lo limita; pero la circunferencia no está en el círculo,

13 "Wittgenstein's Lecture on Ethics", op. cit., p. 5. 
tampoco está más allá de él; está, por así decirlo, en todo el círculo o, mejor dicho, todo el círculo está en ella. De parecida manera, tanto la lógica como el sentido del mundo y el valor no están en el mundo, sino que lo "engloban" todo entero. El sentimiento y visión místicos se refieren justamente a ese todo determinado por el espacio lógico y por la buena o mala voluntad.

Así, para expresar lo místico sólo se emplean oraciones negativas que dicen que no es en el mundo, o bien oraciones que indican que concierne al todo.

Una variante de estas últimas son oraciones que afirman la identidad o la correspondencia entre dos maneras distintas de referirse al mismo todo.

Ejemplos: "Los límites de mi lenguaje se refieren a los límites de mi mundo" (5.6).

"El mundo y la vida son lo mismo" (5.621).

"Yo soy mi mundo" (5.63).

"La ética y la estética son lo mismo" (6.421).

Estas oraciones sólo establecen una identidad o correspondencia entre dos signos que se refieren a condiciones del todo. El aspecto paradójico de algunas de ellas proviene de que podemos tomar los términos identificados como referidos a una parte del todo, a un hecho o conjunto de hechos; resulta entonces extraña su identificación. Por ejemplo, 5.621 y 5.63 parecen absurdos si por "vida" o por "yo" entendemos un conjunto de acontecimientos particulares que suceden en una región del mundo (en mi "individualidad"), pero no lo son si por "yo" y por "vida" se entiende justamente un límite del mundo que abarca todo ("El sujeto... es un límite del mundo", 5.632). Los términos referidos a la totalidad se identifican.

3. Oraciones sobre el método correcto de plantear ciertas preguntas y respuestas.

Ejemplo: "La solución del problema de la vida se hace notar en la desaparición de ese problema" (6.521). 
Estas son muy pocas y no se refieren propiamente a lo indecible sino a las cuestiones que pueden plantearse.

Sólo algunas oraciones, en las partes consideradas, no caen dentro de esos tres tipos y predican alguna cualidad o relación de algo que no está en el mundo. Los únicos casos, creo, serían los siguientes:

6.422: sobre el premio y castigo éticos.

$6.431,6.4311$ y 6.4312 : sobre la muerte y la eternidad.

Salvo por esas contadas excepciones, que yo no acertaría a justificar, ninguna de las oraciones sobre lo indecible consiste en predicar alguna cualidad o relación de ello, ninguna pretende describir cómo sea lo indecible. Así, no se puede decir cómo sea "Dios", o "lo más alto", en qué consista la "buena o mala voluntad", qué se entienda por el "sentido de la vida", etc. Las oraciones de los Notebooks 19141916 que se referían a cosas semejantes y presentaban una forma descriptiva han desaparecido del Tractatus. Lo cual nos hace pensar que Wittgenstein las consideró impertinentes. Las oraciones del Tractatus sobre lo indecible se limitan a tratar de comunicar una de dos: o bien lo que no es lo indecible, o bien que lo indecible se refiere al todo. Pero lo segundo podría, en rigor, reducirse a lo primero: expresar que lo indecible corresponde al todo es sólo decir que no es una parte del todo, que no está en el todo (el mundo). Así, las oraciorres sobre lo indecible se limitan a comunicar siempre lo mismo: lo indecible es de tal manera que no se encuentra en el mundo. No dicen nada más. Para expresar lo indecible sólo cabe la "vía negativa".

Esta observación puede abrirnos una pista para entender cómo pueden comunicar algo esas oraciones.

\section{Clases de sinsentidos}

Pero todas esas oraciones carecen de sentido. Sentido sólo tienen las proposiciones que figuran la realidad y esas oraciones no pueden figurar nada. ¿Qué clase de sinsentido son? Ante todo, hay que distinguir entre dos tipos de sinsell- 
tido que Wittgenstein designa con palabras diferentes: Sinnlos y Unsinnig. El primero se aplica a pseudoproposiciones que forman parte de las reglas lógicas del lenguaje significativo pero que no representan ninguna situación objetiva (Sachverhalt). Las tautologías y las contradicciones son sinnlos (4.461 y 4.462), las leyes de inferencia lo son igualmente (5.132). Sin embargo, las tautologías y las contradicciones no son unsinnig porque pertenecen al simbolismo lógico (4.4611). Las oraciones sinnlos no dicen nada, pero forman parte del lenguaje figurativo y muestran así las propiedades formales del lenguaje y del mundo (6.12).

Unsinnig se aplíca, en cambio, a toda oración que no úsa los signos conforme a las reglas lógicas que hacen posible la formación de figuras. Por lo tanto, ni pueden representar nada ni forman parte del simbolismo mismo. Wittgenstein menciona varias maneras en que estas oraçiones pueden vio. lar las reglas lógicas:

1. Al incluir signos a los que no se les ha dado referencia (Bedeutung) (5.473; cfr. también 4.003).

2. Al usar signos en contra de las reglas que regulan su uso $(4.1272,5.5303,4.1274)$.

3. Al usar signos que hablan de hechos para pretender hablar de proposiciones. Por ejemplo, aseverar que $p$ tiene una propiedad formal o interna (4.124) o que $p$ es una proposición (5.5351).

La mayoría de las cuestiones filosóficas son unsinnig $(4.003,6.53)$. Las oraciones sobre lo indecible, de que hemos hablado, son también sinsentidos en esta acepción.

Sin embargo, no todos los sinsentidos cumplen la misma función. Hay algunos que "elucidan" algo. "Mis proposiciones elucidan (erläutern) de este modo: quien me comprende las reconoce a la postre como sinsentidos, si ha pasado por ellas y ha subido sobre ellas para rebasarlas. (Debe, por así decirlo, tirar la escalera después de haber subido.) Debe superar (überwinden) esas proposiciones, entonces ve el mundo correctamente" (6.54). Si bien esas proposiciones 
carecen de sentido, pueden ser de alguna manera "comprendidas" y, al ser superadas, permiten "ver el mundo correctamente". No pueden ser, por lo tanto, del mismo tipo que "lo bueno es más idéntico que lo bello", citado como ejemplo de oración filosófica sin sentido (4.003). Estamos, pues, obligados a distinguir, dentro de lo unsinnig, entre dos clases de sinsentidos. Llamémoslos simplemente sinsentido ${ }_{1}$ y sinsentido ${ }_{2}$

Al sinsentido ${ }_{1}$ pertenecerian expresiones como "lo para que verde" "el número 3 es amarillo" o "lo bueno es más idéntico que lo bello" Al sinsentido ${ }_{2}$, las oraciones del Tractatus que pretenden hablar de lo indecible y que, de algún modo, serían "elucidativas".

Tanto el sinsentido ${ }_{1}$ como el sinsentido ${ }_{2}$ pecan contra las reglas lógicas que permiten que el lenguaje tenga sentido. Son pues pseudoproposiciones que no figuran la realidad. Sin embargo, el sinsentido ${ }_{1}$ no es en ningún modo comprensible, nada comunica, debe, por ende, simplemente rechazarse. El sinsentido ${ }_{2}$ puede de algún modo comprenderse, comunica algo, debe, por ende, ser usado y luego superado y abandonado.

El sinsentido ${ }_{1}$ viola las reglas particulares de la lógica que señalan cómo debe estar formada una proposición. Lo que pretende decir no sólo no puede decirse sino tampoco mostrarse. El sinsentido ${ }_{2}$ viola la regla más general de la lógica: intenta decir algo que sólo se muestra. Decir lo que se muestra peca contra las reglas de la lógica de una mane. ra peculiar; viola una condición general de toda figura: no se pueden figurar las condiciones de toda figura.

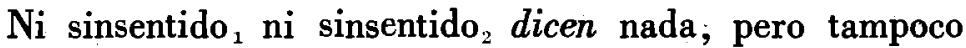
muestran nada. En efecto, sólo las proposiciones con sentido dicen, y sólo en ellas se muestra algo. Sin embargo lo que pretendería decir el sinsentido ${ }_{1}$ no puede mostrarse nunca en ninguna parte, lo que pretende decir el sinsentido ${ }_{2}$ se muestra en otra parte: en la totalidad de las proposiciones con sentido y del mundo al que se refieren. Los sinsentidos ${ }_{2}$ 
pretenden comunicar las condiciones supuestas en el lenguaje con sentido. ¿Cómo pueden éstas mostrarse?

Distinguimos antes entre condiciones de dos niveles dis. tintos: lógicas y extralógicas. De manera correspondiente, podemos distinguir, entre 1 sinsentidos ${ }_{2}$, las pseudoproposiciones de la lógica, que pretenden comunicar las condiciones lógicas de todo lenguaje, y las pseudoproposiciones de la ética y de "lo místico", que indican la condición úl. tima de la lógica misma. El modo como se muestra lo que pretenden comunicar varía en uno $u$ otro tipo de sinsent1$\operatorname{dos}_{2}$ : Veamos primero los sinsentidos 2 de la lógica.

Tomemos dos ejemplos: la pseudoproposición (a) "Los objetos son simples" no muestra que los objetos sean simples. Sólo las proposiciones con sentido muestran ese sentido (4.022) y $(a)$ no es una proposición. Que los objetos sean simples lo deben mostrar otras oraciones, al ser usadas: todas las proposiciones elementales del lenguaje con sentido. "Hay dos objetos" no muestra que haya dos objetos, ésto se muestra en cualquier proposición de la forma $\Phi(a, b) .^{14}$

La pseudoproposición (b) "La lógica es trascendental" tampoco muestra nada. Que la lógica sea trascendental (esto es, que esté supuesta en todo lenguaje) se muestra en el uso de las proposiciones con sentido sujetas a reglas lógicas. Al usar esas proposiciones, se muestra el conjunto de las reglas como condición de toda proposición con sentido. La lógica no es mencionada en esas proposiciones, sólo es usada. Así, los sinsentidos ${ }_{2}$ pretenden mencionar lo que sólo se manifiesta en el uso de proposiciones que tienen otra mención.

Tampoco los sinsentidos ${ }_{1}$ muestran lo que pretenden decir. Por ejemplo, la pseudoproposición (c) "El bien es amarillo" no muestra el color del bien. Pero hay una diferencia entre $(a)$ y $(b)$ por una parte y $(c)$ por la otra. Lo que pretenden mencionar $(a)$ y $(b)$ es mostrado por las propie.

14 Carta a Russell, 19, 8, 19, en Notebooks, p. 130. 
dades del lenguaje al ser usado, lo que pretende mencionar (c) no es mostrado nunca por nada. Lo que quieren decir (a) y (b) corresponde a las condiciones que permiten el uso del lenguaje, lo que quiere decir $(c)$ no corresponde a nada. Los sinsentidos ${ }_{2}$ pretenden justamente decir cuáles son las condiciones supuestas en el lenguaje para figurar un mundo, pero esto sólo el uso de las proposiciones con sentido puede mostrarlo. Pero entonces lo que esos sinsentidos de la lógica pretenden decir debe poderse mostrar en otras oraciones. ${ }^{15}$

Si admitiéramos la posibilidad -que excluye el Tracta. tu - de un lenguaje de segundo nivel que hablara con sentido de lo que se muestra en el lenguaje de primer nivel, los sinsentidos ${ }_{1}$ seguirían careciendo de sentido en ese lenguaje de segundo nivel; los sinsentidos ${ }_{2}$ de la lógica, en cambio, sólo seguirían careciendo de sentido si los tomára. mos como perteneciendo al lenguaje de primer nivel (que es lo que justamente hace el Tractatus, al no admitir len. guaje de segundo nivel), pero podrían ser proposiciones con sentido en el lenguaje de segundo nivel.

Pasemos ahora a los sinsentidos 2 que tratan de lo ético y de lo místico. Lo que pretenden comunicar son condiciones de existencia de las mismas reglas lógicas. Se refieren al supuesto que está debajo, tanto de los proposiciones como de sus condiciones lógicas: la existencia de un mundo como un todo limitado por el espacio lógico. Por ello no esta. rían incluidas en un lenguaje de segundo nivel, que se limi. tara a establecer las reglas sintácticas usadas en el lenguaje de primer nivel. Serían supuestos no mencionados, tanto de ese lenguaje de segundo nivel como de cualquier len.

15 Max Black no tiene razón, en mi opinión, al sostener (op. cit., p. 381)

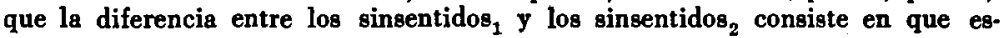
tos últimos muestran lo que quieren decir. Frente a esa tesis, me parece co. rrecta la de P. Hacker (Insight and Illusion, Clarendon Press, London, 1972, pp. 28-29) cuando afirma que los sinsentidos tampoco muestran nada. En efecto, ningún sinsentido puede mostrar; son sólo las proposisiciones las que que pueden mostrar lo que las pseudoproposiciones quisieran decir. 
guaje de cualquier nivel y de cualquier realidad a la que se refiriera el lenguaje.

Al igual que los sinsentidos ${ }_{2}$ de la lógica, los de la ética y lo místico deben poder mostrarse en el lenguaje figurativo. Pero lo ético y lo místico se muestra de un modo enteramente distinto a la forma lógica. Acudamos a la socorrida analogía de un juego reglado, el ajedrez, por ejemplo. Las movidas de las piezas serían, en ese juego, el análogo de las proposiciones en el lenguaje. Un observador podría com. prender, atendiendo a las distintas jugadas, las reglas usadas en el juego. Éstas se muestran en las jugadas, sin necesidad de ser expresadas. Pero si contemplamos el juego como un "todo limitade", constituido por un conjunto de jugadas enlazadas entre sí, dentro del "espacio lógico" de la partida, se puede mostrar también un "sentido" global de la partida, que no está "dicho" en ninguna jugada ni forma parte de las reglas del juego. Para encontrar ese sentido, no es menester buscarlo en "hechos" ajenos a las mismas jugadas, en la psicología de los contendientes, por ejem. plo, sino que puede verse en el conjunto de las jugadas consideradas como un todo coherente. La partida entera revela un propósito y puede mostrar armonía, decisión, belleza, valor, en suma. Sentido y valor pueden ser experimentados en el juego aunque no constituyan movimientos del juego ni tampoco condiciones formales que los regulen. Igual en el lenguaje: sentido y valor pueden ser mostrados por las proposiciones que hablan de hechos, aunque no sean hechos ni puedan expresarse en proposiciones. Pero para ello es menester una visión de la partida, o del lenguaje, que no se limite a detectar sus reglas formales, sino que los contemple como un conjunto de hechos existentes, válido por sí mismo, íntegro y completo, por así decirlo.

Mientras los sinsentidos ${ }_{2}$ de la lógica no implican ninguna "experiencia", salvo la de las proposiciones en uso, los sinsentidos $_{2}$ de la ética y de lo místico expresan una "experiencia" del mundo. Para que las proposiciones muestren 
sus condiciones lógicas no es menester ninguna actitud o sentimiento, para que el mundo y su representación muestren lo ético y lo místico es menester una actitud.

Los sinsentidos, por último, no pueden, en ningún sentido, ser verdaderos ni falsos. Los sinsentidos ${ }_{2}$ - tanto de la logica como de la ética y de lo místico- tampoco son verdaderos ni falsos, puesto que, al no figurar ninguna situación objetiva, no son verificables por ningún hecho. Pero en un sentido peculiar podríamos decir que pueden "comprobarse", en la medida en que en las proposiciones o en el lenguaje y el mundo como un todo se muestre lo que pretenden expresar. Podríamos decir que, si bien no son verdaderos ni falsos, se refieren a las condiciones que hacen posible que cualquier proposición sea verdadera o falsa, las cuales se muestran en la totalidad de las proposiciones y del mundo.

Pero entonces parece que, a diferencia de los sinsentidos, los sinsentidos ${ }_{2}$, aunque no digan ni muestren nada, pueden "comunicar" algo: lo que se muestra en el lenguaje con sen. tido. ¿Cómo es ésto posible? ¿Habrá una "comunicación" distinta al decir y al mostrar?

\section{La comunicación de lo indecible}

En primer lugar, las reglas lógicas son usadas, no mencionadas, en las proposiciones con sentido; se muestran en el uso correcto de los signos. Pero su uso incorrecto revela los límites de aplicación de las reglas y, por ende, las determina. Si no pudiéramos contrastar el uso correcto de un símbolo con contraejemplos de aplicaciones incorrectas, no se delimitarían claramente las reglas. Los sinsentidos, al no estar conformes a las reglas lógicas, no muestran directamente éstas, pero permiten que se vean claramente en las proposiciones, por contraste; indican pues los límites de aplicación de la lógica. Así, las pseudoproposiciones permiten orientar nuestra atención hacia la estructura lógica de las proposiciones, al señalar sus límites de aplicación. 
Un ejemplo del propio Wittgenstein. La palabra "objeto" sólo puede expresarse correctamente por una variable (4.1272). "No se puede, por ejemplo, decir 'hay objetos', como se dice "hay libros'." "¿Por qué entonces aparece esa pseudoproposición ("hay objetos") en esa entrada? Al compararla con la proposición correcta "hay libros", puedo percatarme de que la primera es un sinsentido; al verlo, puedo notar también cuál es el uso correcto del concepto formal "objeto" en las proposiciones con sentido. Así, en cualquier proposición en que se instancie la variable que expresa el objeto, se muestra dicho concepto formal y la regla (no mencionada) que rige su uso. "El concepto formal está dado ya con un objeto que caiga bajo él" (4.12721). El sinsentido ("Hay objetos") ha servido para dirigir nuestra atención a los conceptos, formales que, sin ser mencionados, se muestran en las proposiciones con sentido. Al intentar decir, en ese sinsentido, lo que se muestra en cualquier proposición he llamado la atención justamente sobre aquello que se muestra.

Los sinsentidos no $_{2}$ muestran nada por sí mismos pero orientan al interlocutor para que se fije en lo que el lenguaje le muestra al ser usado correctamente. Al captarse como sinsentidos, delimitan el lenguaje con sentido y hacen posible que, dentro de esos límites, nos fijemos en lo no dicho por el lenguaje. Tendrían la función de un índice que señala lo que el lenguaje y el mundo muestran, para que el otro lo vea por sí mismo. Tratarían de indicar lo que cada quien debe intuir sin palabras.

"Los límites de mi lenguaje se refieren (bedeuten) a los límites de mi mundo" (5.6). Así, las pseudoproposiciones sobre los limites del lenguaje tendrían cierta "referencia". La entrada 4.115 parece corroborarlo: la filosofía, dice, "se referirá (Sie wird ... bedeuten) a lo indecible, al presentar claramente lo decible". Lo indecible puede ser, pues, objeto de referencia. ¿Cómo? Es claro que en esas entradas "referencia" no puede tener el sentido que tiene en el resto 
del libro: la relación entre un nombre y el objeto que representa. En efecto, un signo sólo tiene referencia en la proposición (3.314) v los sinsentidos ${ }_{2}$ no contienen propiamente nombres ni son proposiciones. "Rereferencia" debe, pues, significar aquí algo más vago, como "indicación" o "sugerencia". Ciertos sinsentidos podrían "referir" indirectamente al interlocutor a aquello que pretenden decir, dirigiéndolo para que él vea lo que su lenguaje y su mundo le muestran. "Referir" querría decir aquí: indicarle al otro lo que sólo puede ver por si mismo.

De allí la importancia de la "vía negativa". Sólo si tienen una forma negativa, los sinsentidos ${ }_{2}$ evitan la falacia de pretender figurar una situación objetiva. Una pseudoproposición afirmativa como "Dios es el sentido del mundo" o "el valor depende de la buena voluntad", parecería indicarnos, por su forma, que figura una situación objetiva. Pero esto es imposible, al formularlas, nos incitarían a buscar en el mundo, una situación objetiva inexistente. En cambio, pseudoproposiciones negativas como "Dios no se revela en el mundo" o "En el mundo no hay valor" no pretenden figurar ninguna situación objetiva; pretenden comunicar justamente que algo no se da nunca como componente de una situación objetiva, luego, pueden orientar a ver algo que no veríamos si lo buscáramos en una situación objetiva.

Los sinsentidos: tratan de traducir en palabras lo dado en una visión irrepresentable, al intentarlo fracasan, pero ese fracaso "hace" algo: indica ("refiere") al otro aquello que él puede esperar ver por sí mismo, sin decirlo. Tienen una función pragmática: desviar la atención del otro de los hechos, dirigirla hacia el todo limitado y ponerlo alerta para que pueda ver algo. Sólo en este sentido, podría admitirse que tengan cierta "referencia".

Pero entonces el sinsentido ${ }_{2}$ debe poder señalar lo que debemos buscar en nuestro mundo. Es menester, pues, que de algún modo pueda entenderse lo que el sinsentido "trata de decir" sin lograrlo. En esto se diferenciaría el sin. 
sentido $_{2}$ del sinsentido ${ }_{1}$. Si dijera, por ejemplo, "Bubu no se manifiesta en el mundo", "las proposiciones no pueden expresar bubu" o "bubu es trascendental", estos sinsenti$\operatorname{dos}_{1}$ no me permitirían ver nada. En efecto; el pseudosigno "bubu" no sólo carece de referencia, tampoco me dirige para que busque en mi lenguaje algo que pueda hacerse presente. En cambio, si digo "el objeto es inexpresable", "Dios no se manifiesta en el mundo", "las proposiciones no pueden expresar el valor" o "la ética es trascendental", esos sinsentidos $_{2}$ señalan cuál es la referencia que no puedo darles a los pseudosignos "objeto", "Dios", "valor" y "ética", pero también pueden indicarme, eventualmente, lo que puedo esperar que se muestre "fuera" del mundo, esto es en la totalidad de los hechos y de las proposiciones. Esta diferencia entre un pseudosigno como "bubu" y otros, como "objeto", "Dios", "valor", o "ética", usados en el Tractatus, no la plantea Wittgenstein. No podemos saber si trató siquiera de explicarla. Sólo podemos, por lo tanto, arriesgar alguna hipótesis que pudiera no corresponder al pensamiento del autor.

"Objeto", "Dios", "valor", "vida", etc., tienen un sentido confuso y una referencia vaga en el lenguaje ordinario. Antes de analizar esos signos, nos sirven para referirnos a ciertos hechos o conjuntos de hechos en el mundo. Por ejemplo, "objeto" puede referirse a una cosa percibida, "vida", a un conjunto de acontecimientos espacio-temporales psicofísicos, "Dios" a una entidad ideal o "sobrenatural" de que hablan las religiones, "valor" a alguna cualidad de objetos de mi preferencia, etc. Esta referencia no se establece nunca con precisión. Por ello no pueden ser usados adecuadamen. te. Un breve análisis los mostraría "bedeutunglos" (3.328). A esas palabras del lenguaje cotidiano no llegan a corres. ponderlés, por lo tanto, símbolos precisos (un símbolo es "cualquier parte de la proposición que caracteriza su sentido", 3.31) que contribuyan al sentido de las oraciones de que formen parte. Son pues, estrictamente, sinsentidos. Sin 
embargo, puesto que existe una cierta referencia, por vaga e imprecisa que sea, podríamos admitir que hay una cierta comprensión, igualmente vaga, de las pseudoproposiciones de que forman parte. Si comprender una proposición es "conocer la situación objetiva que representa" (4.021) o "conocer lo que acontece si es verdadera" (4.024), habría una cierta comprensión vaga de algunas pseudoproposiciones, en la medida en que sus signos tuvieran cierta referencia también vaga que permitiera determinar alguna "situación objetiva" confusa. Claro está que para Wittgenstein, no hay situación objetiva si no está determinada, ni hay símbolos si no tienen una referencia precisa. La situación objetiva que "existiría" si la pseudoproposición fuese verdadera, por ser confusa, no puede llamarse propiamente. "situación objetiva". Pero, de hecho, es posible psicológicamente la representación confusa de lo que acontecería si fuesen verdaderas las pseudoproposiciones del lenguaje ordinario donde aparecen términos como "objeto", "Dios", "valor" o "vida", referidos a hechos en el mundo. Luego, es psicológicamente posible una representación confusa de lo que "tratan de decir".

Ahora bien, las oraciones negativas del Tractatus cancelan esa referencia vaga. Comunican que términos como los anteriores no son aplicables a hechos del mundo, luego, no tienen la referencia con que los usábamos en el lenguaje cotidiano. Pero, al mismo tiempo, las oraciones sobre el todo del Tractatus me "refieren" (en el sentido pragmático de me "indican", "dirigen") a otra cosa, sólo que eso ya no es algo en el mundo, sino algo que sólo puede manifestarse en la visión del mundo como un todo y de sus límites. No hay pues, estrictamente, una anulación de la referencia vaga sino una "trasposición" de la misma, de hechos en el mundo a sus límites. Por ejemplo, "objeto" ya no se refiere a cualquier cosa percibida cambiable, sino a la sustancia inalterable (2.071), "vida" ya no se aplica a ciertos acontecimientos psicofísicos en el mundo sino al mundo mismo en 
cuanto contemplado por mí ("El mundo y la vida son uno", 5.621 ); "Dios" ya no designa un ente sobrenatural sino el "sentido del mundo"," "ética" ya no se refiere a oraciones sobre cualidades de las cosas, sino a oraciones sobre "lo más digno de ser vivido". ${ }^{17}$

El mismo Wittgenstein deja notar, en una ocasión, esta trasposición de la referencia. El término "yo" pasa de una referencia vaga al sujeto psicofísico ("el hombre, el cuerpo humano o el alma humana") a la indicación del "sujeto metafísico", que es "el límite del mundo - no una parte de él” (5.641). En esa trasposición de la referencia, cambia el sentido de "referencia". Pasamos de la referencia vaga (en un sentido semántico) de un pseudonombre ("yo") a un objeto o hecho en el mundo ("el hombre"), a la referencia (en un sentido pragmático) a los límites del mundo.

Debemos suponer que, en esa "trasposición", se conserva la comprensión vaga de lo que pretendían decir las palabras en su uso ordinario. Esa comprensión sirve entonces de orientación para buscar, en la visión del mundo como un todo, algo "análogo" que se haga manifiesto. Pero ese "análogo" ya no puede expresarse con las mismas palabras que usaba en el lenguaje ordinario ni es objeto de la misma referencia vaga. Es indecible. Sólo puede captarse directamente por el sentimiento y la intuición sin palabras. Pertenece a la esfera de la voluntad y no a la de la representa. ción. Los sinsentidos ${ }_{2}$ permiten así pasar de las pseudoproposiciones del lenguaje ordinario, con referencia vaga a algo que no puede presentarse, a la visión de algo "análogo" que puede manifestarse, sin representación, ante el sentimiento y la voluntad. Las pseudoproposiciones que trataban de representar lo irrepresentable son como alegorías que apuntan a algo que corresponde al campo de lo no-representable: la voluntad y el sentimiento. Al igual que una alegoría, permiten fijar nuestra atención sobre algo que sólo

16 Notebooks, p. 73.

17 "Wittgenstein's Lecture on Ethics", op, cit., p. 5. 
podemos ver por nosotros mismos, en nuestro mundo. Pero esto sólo lo pueden hacer en la medida en que comprendamos que no representan nada (que carecen de sentido).

Ahora podremos, tal vez, entender mejor la paradoja de 6.54. Los sinsentidos ${ }_{2}$ se "referirían" a lo indecible mediante una triple operación: 1 . Cancelando la rẹferencia vaga a hechos en el mundo de las oraciones análogas del lenguaje ordinario. 2. Transfiriendo esa "referencia" a los límites del mundo y del lenguaje. 3. Dirigiendo así nuestra atención hacia lo que puede mostrarse en nuestro lenguaje y en nuestro mundo. Sólo "al final" (Am Ende), después de haber usado esas pseudoproposiciones "al través de ellas y sobre ellas" ("durch sie - auf ihnen"), nos damos clara cuenta de que carecen de sentido, esto es, de que no pueden representar nada del mundo sino que "hacen" otra cosa. Y sólo al comprenderlas como sinsentidos ${ }_{2}$ podemos acceder a la "visión correcta" del mundo. Porque sólo entonces dejamos de buscar en los hechos representables la referencia de las pseudoproposiciones y se nos puede mostrar algo análogo en el todo irrepresentable. Cumplida esa función, los sinsentidos $_{2}$ ya son inútiles; sólo queda la visión directa del mundo presente ante el sentimiento y la voluntad: "debemos guardar silencio" (7).

\section{La visión "correcta" del mundo}

El Tractatus no elimina la ética ni la metafísica. Antes al contrario, pretende hacerlas posibles liberando de la ilusión que obstruye su comprensión "correcta". La ilusión tradicional de la ética y la metafísica ha sido creer que podemos encontrar en el mundo representable por el lenguaje aquéllo a que se refieren. Mientras no comprendamos claramente que esa via es intransitable (mientras no aceptemos que todo intento de representar lo ético y lo metafísico conduce necesariamente a sinsentidos), no podremos ver el análogo de lo que trataban de comunicarnos. Porque sólo entonces se puede mostrar en la esfera de la voluntad y del sentimiento 
algo similar a lo que las pseudoproposiciones de la ética y la metafísica pretendían indicar en la esfera de la represen. tación.

La renuncia a la vía de la ilusión deja el lugar a la visión del mundo como un todo limitado. En ella se manifiesta lo que la ilusión impedía ver. Así, sólo al comprender que carece de sentido formular cualquier proposición sobre el "sentido de la vida", dejo de buscar afanosamente ese sen. tido en algún acontecimiento del mundo, por ejemplo, en la realización de una obra, en la obtención de algún bien o en el logro de algún objetivo, porque sólo entonces comprendo que el "sentido de la vida" no se refiere a ningún hecho. $\mathrm{Y}$ sólo entonces estoy en disposición de captar, en la totalidad de la vida, su sentido, porque comprendo que éste no se encuentra en ningún acontecimiento de la vida, sino en la vida misma: el sentido de la vida es vivirla en plenitud ("Cumple el propósito de la existencia quien ya no necesita ningún propósito salvo vivir."). ${ }^{18}$

De parecida manera, al comprender que Dios no se manifiesta en el mundo, dejo de buscarlo en alguna entidad sobrenatural, por más "alta" que sea, y renuncio a describirlo con palabras, como si fuera "algo". Sólo entonces estoy en franquía para que se me muestre lo Sagrado en el estupor que me produce el milagro incomprensible de la existencia del universo. Sólo entonces puedo captar que Dios no es algo distinto del mundo o de la vida sino el sentido mismo de ambos. ("El sentido de la vida, i.e. el sentido del mundo, podemos llamarlo Dios"; "Creer en Dios es ver que la vida tiene un sentido". ${ }^{10}$

Así también mientras no me dé cuenta de que el bien y el mal no se encuentran en el mundo, como cualidades de los hechos, los buscaré en el cumplimiento de determinadas acciones y trataré de expresarlos en principios morales generales. Sólo cuando comprenda que bien y mal —al igual

18 Notebooks, p. 73.

19 Ibid., pp. 73, 74. 
que dicha y desdicha - no son cualidades ni consecuencias de ninguna acción en el mundo, captaré el bien y el mal en la actitud de la voluntad hacia el mundo y en la plenitud o estrechez con que éste se me presente. Sólo entonces podré estar en paz, aceptar plenamente el mundo, "estar en concordancia" con él; sólo entonces estaré en posibilidad de ser verdaderamente bueno y dichoso. ("Para vivir dichoso debo estar en concordancia con el mundo"; "Simplemente la vida dichosa es buena, la desdichada, mala".) ${ }^{20}$

La comprensión de que todo el Tractatus desemboca en pseudoproposiciones, libera de la ilusión de encontrar el valor y el sentido en hechos representables del mundo y franquea así la visión correcta del sentido y del valor del mundo y de la vida, que se manifiestan en la existencia del mundo y de la vida mismas. $Y$ esto no puedo ya captarlo mediante el pensamiento. Debo poderlo vivir, sin palabras, en el sentimiento del "milagro" del universo y en la conformidad de mi voluntad con él. Comprender que las preguntas metafísicas carecen de sentido es la única vía para captar por mí mismo aquello por lo cual preguntan.

Al restringir el lenguaje con sentido a las proposiciones de la ciencia, el Tractatus elimina radicalmente a la ética y a la metafísica de la esfera de la representación y, por ende, del pensamiento. Esa postura coincide con un "positivismo radical". Pero esa eliminación no tiene por función suprimir la ética ni la metafísica, sino abrir la posibilidad de la única metafísica plenamente coherente con esa postura "positivista": la que puede mostrarse "fuera" de la esfera del pensamiento. Parodiando a Kant, podríamos decir que el Tractatus quiso poner límites al pensamiento, para hacer lugar al sentimiento y a la voluntad.

20 lbid., p. 75, 78. 
The Tractatus aims at establishing the ultimate conditions which make possible a meaningful language. These conditions, however, cannot be said within that language. Thus, the book takes the reader from the analysis of what can be said to its inexpressible conditions. These inexpressible conditions are the limits of the world.

This paper deals with the inexpressible limits of language and of the world. Three questions are raised:

(i) How does the Tractatus lead to the view of the world as a limited whole?

(ii) How is it possible to communicate such a view by means of meaningless expressions?

(iii) Which is the "right" view of the world that such a communication make possible?

\section{The conditions of what can be said}

The analysis of the meaningful language shows certain inexpressible conditions that make it possible.

First, it makes one accept the ultimate elements which are represented in propositions by simple signs, i.e. objects. Objects cannot be put into words, they are inexpressible.

Second, it leads to the admission of logical form as an a priori condition of language. Logical form is also inexpressible, but it is shown in every proposition.

Third, it leads us to assume an ultimate extralogical presupposition of the two logical assumptions above mentioned, i.e., the absolutely contingent existence of the world as a whole limited by logical space.

To view the world as a limited whole, (ultimate assumption of all representation) doesn't correspond to the realm of representation but to that of feeling and the will. It is involved in all religious, ethical or esthetical "experience", in all question concerning the "sense" and "value" of the world and of life. To view the world as a contingent and limited whole is the end to which the Tractatus is directed, but it is also its antecedent. Indeed, the entry 1 of the Tractatus presupposes it; the point of arrival is also the point of departure. 


\section{Pseudo-propositions about the inexpressible}

How are we to express this view of the world? Some seemingly unjustifiable exceptions apart, the pseudo-propositions of the Tractatus that talk about the inexpressible are of one of two clases:

(i) Negative sentences. They intend to say that something is not in the world or that a pseudo-proposition is not a part of the language.

(ii) Sentences about the whole or about the limits of the world and of language. They intend to say that something outside the world and the language is shown when the world and the language are viewed as a whole.

The pseudo-propositions of the Tractatus about the inexpressible do not intend to predicate anything of it; they do not intend to describe what the inexpressible is like. They only communicate one and the same thing, i.e. that the inexpressible is such that it does not belong to the world (that it is not a fact) and cannot be described (by means of the picture of a fact). Thus, in order to express the inexpressible the Tractatus turns to the via negationis.

\section{Kinds of nonense}

These pseudo-propositions, however, lack meaning. Two kinds of nonsense should be distinguished. We will call them nonsense ${ }_{1}$ and nonsense $e_{2}$. Both violate the logical rules that make possible a meaningful language. Nevertheless, as nonsense ${ }_{1}$ does not communicate anything, it just has to be rejected. Nonsense ${ }_{2}$, on the contrary, can communicate something; it has to be used and, only later on abandoned. The pseudo-propositions of the Tractatus about the inexpressible are examples of nonsense ${ }_{2}$.

No nonsense says or shows anything. What nonsensical ${ }_{1}$ sentences intend to say is never shown anywhere; what nonsensical ${ }_{2}$ sentences intend to say is shown somewhere else, in the totality of the propositions and of the world. How?

We must distinguish two kinds of nonsense ${ }_{2}$ : those that intend to communicate the logical conditions of language, and those that intend to communicate its extralogical conditions.

The nonsensical ${ }_{2}$ sentences of logic intend to mention the conditions assumed by language, and this is shown only by the use of propositions. The meaningful sentences show what nonsensical ${ }_{2}$ pseudo-propositions of logic cannot say nor show, but what they intend to communicate.

The nonsensical ${ }_{2}$ sentences that intend to express the extralogical conditions are those about ethics and the "mystical". They are shown 
in a different way than logical conditions, i.e. in the view of the world as a limited and contingent whole which is offered, not to thought, but to feeling and the will.

Then, nonsensical ${ }_{2}$ sentences intend communicating what is shown elsewhere, i.e. in the meaningful language and in the view of the world as a whole. There must be, therefore, a kind of communication that is different from saying or showing.

\section{Communication of the inexpressible}

Nonsensical $_{2}$ sentences neither say nor show anything, they orientate the reader in order that he takes notice of what his language and his world show to him. They have the role of pointing out to him what he should see by himself. Nonsensical ${ }_{2}$ sentences "refer" to the limits of the language and the world; but "reference" (Bedeutung) has, in this context, a pragmatic, not a semantical sense.

From this, the importance of the via negationis is clear. The negative pseudo-propositions do not intend to express any state of affairs; they "do" something else. i.e. they divert our atention from states of affairs and point, in the totality of our experience, to something that cannot be presented in any fact.

However, we have to admit a certain vague understanding of what nonsensical ${ }_{2}$ sentences claim to communicate so that they can accomplish that pragmatic role.

The nonsensical ${ }_{2}$ sentences of the Tractatus use pseudo-signs of ordinary language that have, in that language, a confused or void reference. That vague reference (in the semantical sense) is canceled in the nonsensical sentences ${ }_{2}$ of the Tractatus and at the same time they point (in a pragmatic sense) to something analogous (to the vague reference) that can be given in the view of language and of the world as a whole. They direct our attention in order that we see by ourselves something "analogous" to what the vague expressions of ordinary language claim to express, but they can do that only in so far as we understand that they do not represent anything, that they lack meaning. Only by understanding that they are nonsense our attention is directed to what otherwise we would not see, because only then the describable states of affairs are put aside. In that way they let us get a "right" view of the world.

\section{The "right" view of the world}

The Tractatus rejects neither ethics nor metaphysics, it liberates us from the illusion that obstructs their "right" interpretation. The 
traditional illusion of ethics and metaphysics has been to believe that we can find their object in the world that can be pictured by language. So long as we do not understand that that way is not open to us we cannot see by ourselves the analogon of what they tried to communicate. Only then it can be shown in the realm of feeling and the will something similar to what the pseudo-propositions of ethics and metaphysics intended to refer to in the realm of representation.

The understanding that all the Tractatus flows towards nonsense frees one from the traditional illusion of metaphysics and opens the correct view, i.e. we will not try to find sense and value in facts of the world but in the existence of the world and of life itself. I have to live "sense" and "value" in the feeling of the "miracle" of the existence of the world and in the conformity of my will to it. To understand that metaphysical questions are meaning. less is the only way to understand by ourselves the problems with which they are concerned.

When the Tractatus restricts the meaningful language to the propositions of science, ethics and metaphysics are eliminated from the realm of representation and therefore from the realm of thought. But this opens at the same time the possibility of the only metaphysics compatible with that attitude, i.e. the one that can be shown "outside" the realm of thought, in feeling and the will. 